\title{
2D and 3D Simulation of Elastic Wave Propagation by Using Element Free Galerkin's Method
}

\author{
Masafumi Katou $^{1}$, Toshifumi MATSUOKA ${ }^{1}$, Hitoshi MIKADA ${ }^{1}$, \\ Yoshinori SANADA ${ }^{2}$ and Yuzuru ASHIDA ${ }^{1}$ \\ ${ }^{1}$ Dept. of Civil and Earth Res. Eng., Kyoto University \\ ${ }^{2}$ CDEX, Japan Agency of Marine Science and Technology.
}

\begin{abstract}
The element free Galerkin's (EFG) technique is proposed for solving 2D and 3D elastic wave equation. In this paper, we compare the waveforms calculated by three methodologies; Finite Difference Method with 4th order accuracy in space (FDM4), traditional FEM and EFG. Traditional FEM and EFG are tested on two individual interpolating functions (IFUNC); 1st order (plane) IFUNC and 2nd order (curved) IFUNC. From the comparison to the exact waveforms of Lamb's problem, the proposed algorism gives accurate waveforms. By two-dimensional implementation, FEM4 demonstrates more accurate result than traditional FEM with plane IFUNC and EFG with plane IFUNC demonstrates more accurate one than FEM4. However, by employing curved IFUNC, traditional FEM and EFG demonstrate almost same accuracy. Moreover, we expand 2D program to 3D one. On the case of 1st order (plane) interpolating function, EFG improves numerical accuracy of traditional FEM without the increase of computational memory.
\end{abstract}

\section{INTRODUCTION}

The EFG (Element Free Galerkin's method) by Belytschko, et al. (1994) can be useful methodology in terms of that it uses low-order interpolation (second or less). EFG has been applied to dynamic problems such as elastic wave propagation (Lu, et al. 1995; Jia and $\mathrm{Hu}, 2006$ ), and it is expected to perform with high accuracy even using a low-order base function. However, practical accuracy of EFG has not been tested by these early works.

Among various numerical methods to solve the elastic wave equation, FDM(Finite Difference Method) using the staggered grid scheme (e.g. Virieux, 1986; Graves, 1996) may be the most popular method because of its simple coding and satisfactory accuracy. On the other hand, with rapid advancing of computational instruments, SEM (Spectral Element Method) by Komatitsch and Tromp (1999), or ADER-DG (Arbitrary high-order DERivatives Discontinuous Galerkin finite element method) by Dumbser and Kaser (2006) may be considered as the most powerful methodologies since their accuracies are 100 times or more than FDM. Both SEM and ADER are traditional FEM (Finite Element Method) with high-order (fourth or more) interpolation. However, high-order interpolation needs many number of nodal points to define a single element.

When EFG is considered as practical use, FDM with 4th order accuracy in space (FDM4) and traditional FEM are the most serious competitions. In this paper, we compare the waveforms calculated by FDM4, traditional FEM and EFG.

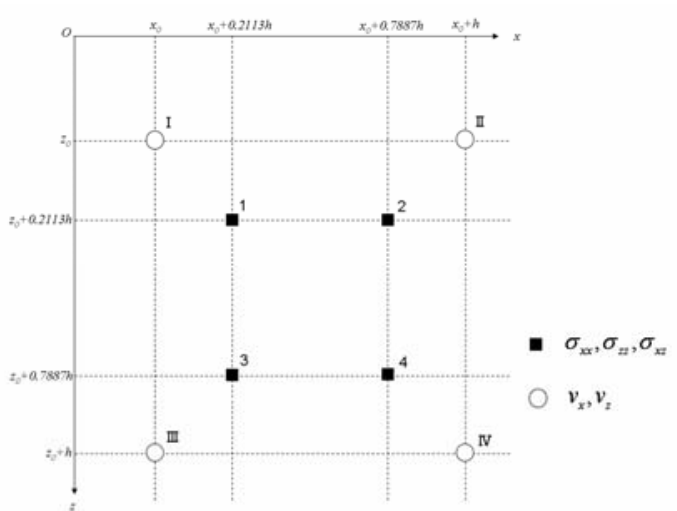

Figure. 1 Grid arrangement for element free Galerkin's scheme in case of 1 st order base and $2 \times 2$ Gauss-Legendre integral points.

\section{METHODOLOGY}

\section{(1) Interpolating shape function}

In element free Galerkin's technique, the velocity vector and the stress tensor are arranged as shown in Fig. 1. This is the simplest case. In the case of a square node, $h$ is the distance between nodes and there are $2 \times 2$ Gauss-Legendre (GL) integral points shown by black squares. The square nodes $(j=\mathrm{I}$, II, III, IV) are represented by open circles in Fig. 1. 
When particle velocity vector is known at these 4 nodes, stress tensor is evaluated at 4 GaussLegendre integral points ( $i=1,2,3,4)$ with multiplying a coefficients to velocity vectors. This coefficient matrix is related to $4 \mathrm{GL}$ points and 4 nodal points and is obtained by element free Galerkin's method as follows.

We will discuss the case of 1st order shape function and then we expand this to the 2nd order case. First of all, let us assume the basis vector of a shape function is as

$$
\begin{array}{cc}
\mathbf{P}^{\mathrm{T}}=[1, x, z] & \text { (1st order) } \\
\mathbf{P}^{\mathrm{T}}=\left[1, x, z, x z, x^{2}, z^{2}, x^{2} z^{2}\right] & \text { (2nd order) }
\end{array}
$$

In this paper, we use the following weight function.

$$
w_{i}\left(r_{i j}\right)=n \frac{r_{i j}}{\mathfrak{R}_{i}}\left(1-\frac{r_{i j}}{\mathfrak{R}_{i}}\right)^{n-1}+\left(1-\frac{r_{i j}}{\mathfrak{R}_{i}}\right)^{n} .
$$

Where $r_{i j}$ is the distance between each pare of the GL point and nodal point. $\mathrm{R}_{i}$ is affection radius for each GL point and $n$ is an arbitrary natural number

In the case of 1st order shape fuction (1a), When a GL point $i$ in an element is located at a point $\left(x_{i}, z_{i}\right)$, the displacement $u_{i}$ can be obtained using the coefficients of the interpolated variable $a_{k}$,

$$
u_{i}=a_{0}+a_{1} x_{i}+a_{2} z_{i} .
$$

The coefficients $a_{k}$ satisfy the following equation:

$$
W_{i} B A=U \text {. }
$$

Where,

$$
\begin{aligned}
& \operatorname{diag}\left[W_{i}\right]=\left[\begin{array}{llll}
w_{i}\left(r_{i \mathrm{I}}\right) & w_{i}\left(r_{i \mathrm{II}}\right) & w_{i}\left(r_{i \mathrm{III}}\right) & w_{i}\left(r_{i \mathrm{~V}}\right)
\end{array}\right]^{\mathrm{T}}, \\
& B=\left[\begin{array}{ccc}
1 & x_{0} & z_{0} \\
1 & x_{0}+h & z_{0} \\
1 & x_{0} & z_{0}+h \\
1 & x_{0}+h & z_{0}+h
\end{array}\right], \\
& A=\left[\begin{array}{lll}
a_{1} & a_{2} & a_{3}
\end{array}\right]^{\mathrm{T}}, \\
& U=\left[\begin{array}{llll}
u(\mathrm{I}) & u(\mathrm{II}) & u(\mathrm{III}) & u(\mathrm{IV})
\end{array}\right]^{\mathrm{T}} .
\end{aligned}
$$

Solving Eq. (4) by moving least square method (e.g. Menke, 1989):

$$
A=\left[B^{\mathrm{T}} W_{i} B\right]^{-1} B^{\mathrm{T}} W_{i} U
$$

Thus Eq. (3) is described as

$$
u_{i}=P_{i} U
$$

where

$$
P_{i}=\left[\begin{array}{lll}
1 & x_{i} & z_{i}
\end{array}\right]\left[B^{\mathrm{T}} W_{i} B\right]^{-1} B^{\mathrm{T}} W_{i}
$$

All $P_{i}$ Vectors for every GL points make a following coefficient matrix:

$$
\Phi=\left[\begin{array}{c}
P_{1} \\
P_{2} \\
P_{3} \\
P_{4}
\end{array}\right]=\left[\begin{array}{cccc} 
& \vdots & & \\
\cdots & \phi_{i j} & \cdots & \cdots \\
& \vdots & & \\
& \vdots & &
\end{array}\right]
$$

This $\phi_{i j}$ is known as a shape function in Element Free Galerkin's method.

\section{(2) Equations of motion}

The partial derivatives of variable $u_{i}$ on each GL point can be calculated by using $\phi_{i j}$ :

$$
\begin{aligned}
& \frac{\partial u_{i}}{\partial x}=\frac{\partial P_{i}}{\partial x} U, \\
& \frac{\partial u_{i}}{\partial z}=\frac{\partial P_{i}}{\partial z} U .
\end{aligned}
$$

Where,

$$
\begin{aligned}
& \frac{\partial P_{i}}{\partial x}= {\left[\begin{array}{lll}
0 & 1 & 0
\end{array}\right]\left[B^{\mathrm{T}} W_{i} B\right]^{-1} B^{\mathrm{T}} W_{i} } \\
&+\left[\begin{array}{lll}
1 & x_{i} & z_{i}
\end{array}\right]\left[B^{\mathrm{T}} \frac{\partial W_{i}}{\partial x} B\right]^{-1} B^{\mathrm{T}} W_{i} \\
&+\left[\begin{array}{lll}
1 & x_{i} & z_{i}
\end{array}\right]\left[B^{\mathrm{T}} W_{i} B\right]^{-1} B^{\mathrm{T}} \frac{\partial W_{i}}{\partial x} \\
& \frac{\partial P_{i}}{\partial z}=\left[\begin{array}{lll}
0 & 0 & 1
\end{array}\right]\left[B^{\mathrm{T}} W_{i} B\right]^{-1} B^{\mathrm{T}} W_{i} \\
&+\left[\begin{array}{lll}
1 & x_{i} & z_{i}
\end{array}\right]\left[B^{\mathrm{T}} \frac{\partial W_{i}}{\partial z}\right]^{-1} B^{\mathrm{T}} W_{i} \\
&+\left[\begin{array}{lll}
1 & x_{i} & z_{i}
\end{array}\right]\left[B^{\mathrm{T}} W_{i} B\right]^{-1} B^{\mathrm{T}} \frac{\partial W_{i}}{\partial z} \\
& \frac{\partial W_{i}}{\partial x}=\frac{\partial W_{i}}{\partial r_{i j}} \frac{\partial r_{i j}}{\partial x}, \\
& \frac{\partial W_{i}}{\partial z}=\frac{\partial W_{i}}{\partial r_{i j}} \frac{\partial r_{i j}}{\partial z} \cdot
\end{aligned}
$$

In the case of dynamic problems such as the elastic wave propagation, the deformation of medium is negligible since the displacement caused by the wave is small. Therefore $\partial P_{i} / \partial x$ and $\partial P_{i} / \partial z$ can be considered as constant throughout the simulation, and need only be computed once, after the geometrical parameter $h$ is given.

As is well known, an elastic wave equation is comprised by two sets of equations: stress-strain relations and the equations of motion. The stress-strain relations are:

$$
\begin{gathered}
\frac{\partial \sigma_{x x}}{\partial t}=(\lambda+2 \mu) \frac{\partial v_{x}}{\partial x}+\lambda \frac{\partial v_{z}}{\partial z} \\
\frac{\partial \sigma_{z z}}{\partial t}=\lambda \frac{\partial v_{x}}{\partial x}+(\lambda+2 \mu) \frac{\partial v_{z}}{\partial z} \\
\frac{\partial \sigma_{x z}}{\partial t}=\mu\left(\frac{\partial v_{x}}{\partial z}+\frac{\partial v_{z}}{\partial x}\right)
\end{gathered}
$$


Where $\lambda$ and $\mu$ are Lame's moduli. $\sigma_{x x}, \sigma_{z z}$ and $\sigma_{x z}$, are the stresses. $v_{x}$ and $v_{z}$ are particle velocities. Employing explicit discretization of second order accuracy in time and interpolation of particle velocity, at a point $\left(x_{i}, z_{i}\right)$, Eqs. (19a) to (19c) become:

$$
\begin{aligned}
& \frac{\sigma_{x x}^{t+T / 2}\left(x_{i}, z_{i}\right)-\sigma_{x x}^{t-T / 2}\left(x_{i}, z_{i}\right)}{T}=(\lambda+2 \mu) \frac{\partial P_{i}}{\partial x} V_{x}^{t}+\lambda \frac{\partial P_{i}}{\partial z} V_{z}^{t},(20 \mathrm{a}) \\
& \frac{\sigma_{z z}^{t+T / 2}\left(x_{i}, z_{i}\right)-\sigma_{z z}^{t-T / 2}\left(x_{i}, z_{i}\right)}{T}=\lambda \frac{\partial P_{i}}{\partial x} V_{x}^{t}+(\lambda+2 \mu) \frac{\partial P_{i}}{\partial z} V_{z}^{t},(20 \mathrm{~b}) \\
& \frac{\sigma_{x z}^{t+T / 2}\left(x_{i}, z_{i}\right)-\sigma_{x z}^{t-T / 2}\left(x_{i}, z_{i}\right)}{T}=\mu\left(\frac{\partial P_{i}}{\partial z} V_{x}^{t}+\frac{\partial P_{i}}{\partial x} V_{z}^{t}\right) \cdot(20 \mathrm{c})
\end{aligned}
$$

Where $T$ is the sampling time length, superscript is time on computing, and

$$
V_{l}=\left[\begin{array}{llll}
v_{l}(\mathrm{I}) & v_{l}(\mathrm{II}) & v_{l}(\mathrm{III}) & v_{l}(\mathrm{IV})
\end{array}\right]^{\mathrm{T}}, l=x, z .
$$

\section{(3) Equations of motion}

The equations of motion are:

$$
\begin{aligned}
& \rho \frac{\partial v_{x}}{\partial t}=f_{x}+\frac{\partial \sigma_{x x}}{\partial x}+\frac{\partial \sigma_{x z}}{\partial z}, \\
& \rho \frac{\partial v_{z}}{\partial t}=f_{z}+\frac{\partial \sigma_{x z}}{\partial x}+\frac{\partial \sigma_{z z}}{\partial z} .
\end{aligned}
$$

Where $f_{x}$ and $f_{z}$ are the components of external force vector and $\rho$ is material density. These partial differential equations give the following weighted residual equations:

$$
\begin{gathered}
\int_{\Omega} \varphi\left(\rho \frac{\partial v_{x}}{\partial t}-f_{x}-\frac{\partial \sigma_{x x}}{\partial x}-\frac{\partial \sigma_{x z}}{\partial z}\right) d \Omega=0 \\
\int_{\Omega} \varphi\left(\rho \frac{\partial v_{z}}{\partial t}-f_{z}-\frac{\partial \sigma_{x z}}{\partial x}-\frac{\partial \sigma_{z z}}{\partial z}\right) d \Omega=0 .
\end{gathered}
$$

In Galerkin's method, the shape function $\phi_{i j}$ in Eq.(12) is used as the weigh function $\varphi$.

\section{(4) Perfectly matched layer}

To apply the PML absorbing boundary condition (e.g. Collino and Tsogka, 2001, Festa and Nielsen, 2003), Eqs.(19a) to (19c) are divided in the following way

$$
\begin{aligned}
& \frac{\partial \sigma_{x x}^{x}}{\partial t}+d^{x^{\prime}} \sigma_{x x}^{x}=(\lambda+2 \mu) \frac{\partial v_{x}}{\partial x}, \\
& \frac{\partial \sigma_{x x}^{z}}{\partial t}+d^{z^{\prime}} \sigma_{x x}^{z}=\lambda \frac{\partial v_{z}}{\partial z}, \\
& \frac{\partial \sigma_{z z}^{x}}{\partial t}+d^{x^{\prime}} \sigma_{z z}^{x}=\lambda \frac{\partial v_{x}}{\partial x}, \\
& \frac{\partial \sigma_{z z}^{z}}{\partial t}+d^{z^{\prime}} \sigma_{z z}^{z}=(\lambda+2 \mu) \frac{\partial v_{z}}{\partial z}, \\
& \frac{\partial \sigma_{x z}^{x}}{\partial t}+d^{x^{\prime}} \sigma_{x z}^{x}=\mu \frac{\partial v_{z}}{\partial x},
\end{aligned}
$$

$$
\frac{\partial \sigma_{x z}^{z}}{\partial t}+d^{z^{\prime}} \sigma_{x z}^{z}=\mu \frac{\partial v_{x}}{\partial z} .
$$

Where,

$$
\begin{aligned}
& \sigma_{x x}=\sigma_{x x}^{x}+\sigma_{x x}^{z}, \\
& \sigma_{z z}=\sigma_{z z}^{x}+\sigma_{z z}^{z}, \\
& \sigma_{x z}=\sigma_{x z}^{x}+\sigma_{x z}^{z},
\end{aligned}
$$

This splitting is called directional splitting. Thus two-dimensional PML needs double the memory cost of non PML case. The damping functions are given by:

$$
\begin{aligned}
& d^{x^{\prime}}=\frac{3 V_{p}}{2 D} \log \left(\frac{1}{R}\right)\left(\frac{x^{\prime}}{D}\right)^{2}, \\
& d^{z^{\prime}}=\frac{3 V_{p}}{2 D} \log \left(\frac{1}{R}\right)\left(\frac{z^{\prime}}{D}\right)^{2} .
\end{aligned}
$$

Where $x$ ' and $z$ ' are distance to PML from the boundary between PML and non PML area for each direction $x$ and $z$ respectively. $D$ is width of PML. $V_{p}$ is the P-wave velocity, $R$ is the theoretical reflection coefficient after discretization, which can be chosen to be a very small number (e.g. 0.001, which is the value used in this paper). For numerical computations, Eqs. (26a) to (26f) are discretized as same procedure that Eqs. (19) become Eqs.(20). The term $d \sigma$ is evaluated by the average value between old time and new time, thus:

$$
d \sigma=d \frac{\sigma^{t+T / 2}+\sigma^{t-T / 2}}{2} .
$$

Equations of motion are also adopted PML, Eqs.(22) and (23) becomes:

$$
\begin{aligned}
& \rho\left(\frac{\partial v_{x}^{x}}{\partial t}+d^{x^{\prime}} v_{x}^{x}\right)=f_{x}^{x}+\frac{\partial \sigma_{x x}}{\partial x}, \\
& \rho\left(\frac{\partial v_{x}^{z}}{\partial t}+d^{z^{\prime}} v_{x}^{z}\right)=f_{x}^{z}+\frac{\partial \sigma_{x z}}{\partial z}, \\
& \rho\left(\frac{\partial v_{z}^{x}}{\partial t}+d^{x^{\prime}} v_{z}^{x}\right)=f_{z}^{x}+\frac{\partial \sigma_{x z}}{\partial x}, \\
& \rho\left(\frac{\partial v_{z}^{z}}{\partial t}+d^{z^{\prime}} v_{z}^{z}\right)=f_{z}^{z}+\frac{\partial \sigma_{z z}}{\partial z} .
\end{aligned}
$$

Where,

$$
\begin{gathered}
v_{x}=v_{x}^{x}+v_{x}^{z}, \\
v_{z}=v_{z}^{x}+v_{z}^{z}, \\
f_{x}=f_{x}^{x}+f_{x}^{z} \\
f_{z}=f_{z}^{x}+f_{z}^{z} .
\end{gathered}
$$




\section{TWO-DIMENSIONAL CASE}

A homogeneous halfspace model of $\mathrm{Vp}=1732 \mathrm{~m} / \mathrm{s}$, Vs $=1000 \mathrm{~m} / \mathrm{s}$ and $\rho=1500 \mathrm{~kg} / \mathrm{m}^{3}$ is employed to test the numerical accuracy of EFG. We can obtain an exact waveforms of Lamb's problem by Saito (1993). Ricker wavelet of peak frequency $50 \mathrm{~Hz}$ is used for source time series. The maximum frequency of Ricker wavelet becomes about $125 \mathrm{~Hz}$ and the minimum wave velocity of both models is $1000 \mathrm{~m} / \mathrm{s}$, therefore, the shortest wavelength becomes $8 \mathrm{~m}$. Thus, we define grid spacing $h=1 \mathrm{~m}$. We have 8 or more grids for the shortest wavelength.

Fig.2 shows waveforms calculated by FDM4, FEM and EFG. The offset distance from the source point is $200 \mathrm{~m}$. Dotted line shows exact waveform by Saito (1993). FEM and EFG are adopted 1st order (plane) interpolating function. EFG gives the most accurate resolution.

Fig.3 shows waveforms calculated by FEM and EFG. The offset distance from the source point is $1000 \mathrm{~m}$. Dotted line shows exact waveform. FEM and EFG are adopted 2nd order (curved plane) interpolating function. Traditional FEM gives little more accurate waveform than EFG.

In case of 1st order basis vector Eq. (1a), the numerical accuracy computed by traditional FEM is poorer than FDM4. Interpolation by moving least square of EFG elaborately improves the numerical accuracy of traditional FEM. In case of 2nd order basis vector Eq. (1b), the numerical accuracies of EFG and FEM are almost same. By using 2nd order basis vector, the numerical accuracy becomes at least five times or more than the case of 1st order basis vector.

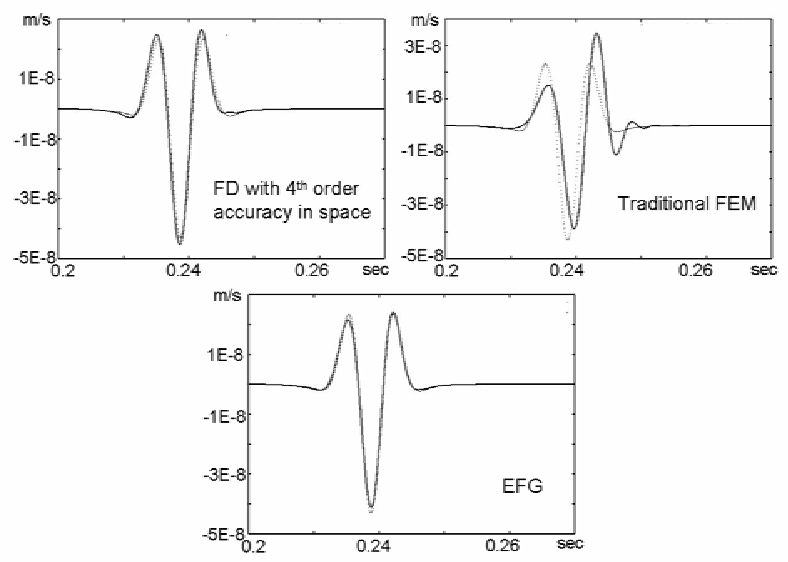

Figure.2 Waveforms calculated by FD, FEM and EFG. The offset distance from the source point is $200 \mathrm{~m}$. Dotted line shows exact waveform by Saito (1993). Both FEM and EFG are adopted 1st order (plane) interpolating function.
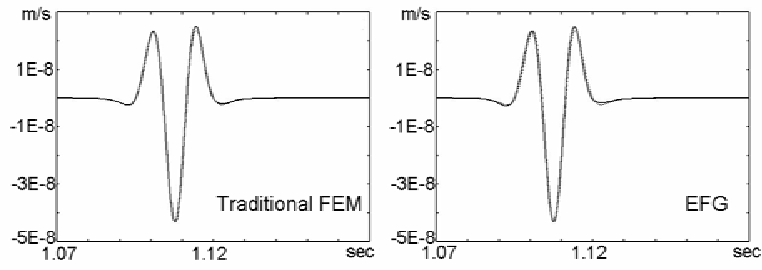

Figure.3 Waveforms calculated by FD, FEM and EFG. The offset distance from the source point is $1000 \mathrm{~m}$. Dotted line shows exact waveform. Both FEM and EFG are adopted 2nd order (curved plane) shape function.
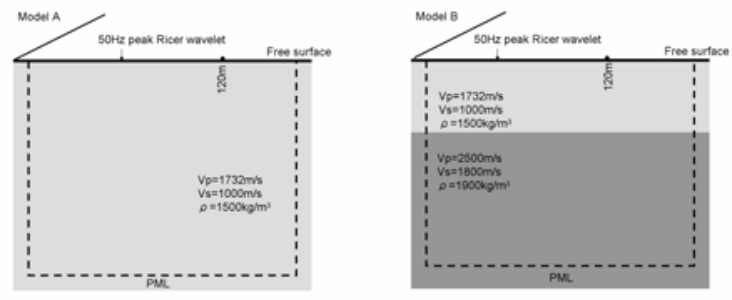

Figure.4 Calculation models

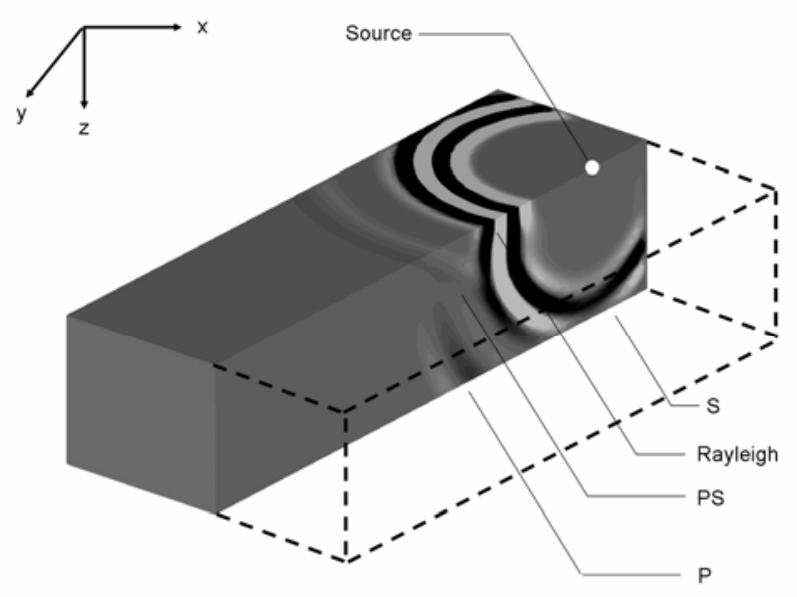

Figure.5 Snapshot of the simulated wavefield.

\section{THREE-DIMENSIONAL CASE}

Two symbolic models are used in order to test the performance of EFG. The two models are shown in Fig.4. Model A is a homogeneous halfspace. We can obtain exact waveforms by Saito (1993). Model B is a two layered halfspace. Although we can not obtain an exact response, reflectivity method supplies semi-analytic waveforms.

We use the following basis vector for threedimensional implementation. 


$$
\begin{aligned}
\mathbf{P}^{\mathrm{T}}= & {\left[1, x, y, z, x y, x z, y z, x^{2}, y^{2}, z^{2} \quad\right. \text { (2nd order) }} \\
& \left., x^{2} y^{2}, x^{2} z^{2}, y^{2} z^{2}, x^{2} y^{2} z^{2}\right]
\end{aligned}
$$

At first, the numerical results for Model A are discussed. Fig.5 shows a snapshot. Top face is free surface. Four side and bottom surface is applied PML absorbing boundary. We can recognize P-wave, S-wave, Rayleigh wave and PS converted wave clearly and PML effectively works as non reflection boundary. Fig.6 shows the calculated waveforms at $120 \mathrm{~m}$ away from source point. EFG is compared to FD (=FDM4) with fourth order accuracy in space. EFG gives little more exact waveform than FD.

Subsequently, model B are discussed. Fig.7 shows the calculated waveforms at $120 \mathrm{~m}$ away from source point. EFG is compared to FD. EFG gives little more exact waveform than FD.

We could not conduct more effective test than Fig. 6 and 7 because of heavy computational costs of EFG. When we calculate the elastic body structured by $161 \times 321 \times 81$ grid points and 8000 steps in time, 40 hours are needed by 8 nodes PC cluster (Pentium IV-3.2GHz and 1Gbps LAN). To speed computational time up is one of the future studies.

\section{Conclusions}

In this paper, Element Free Galerkin's method is used to solve the elastic wave propagation problems. In the simulation test, we assumed that 8 girdpoints would be needed to accommodate the shortest wavelength. The seismogram calculated by EFG is more accurate than FDM with 4th order accuracy in space. We conclude that, on the case of 1 st order (plane) interpolation, EFG improves numerical accuracy of traditional FEM without the increase of computational memory.

\section{ACKNOWLEDGEMENTS}

We appreciate the advice and encouragement from Dr. Jun Ichi, Takekawa; Geo Research Institute and Dr. Jamhir Safani; Kyoto University.

\section{REFERENCES}

1) Belytschko, T., Lu, Y. Y. and Gu. L. (1994). Element-free Galerkin method, Int. J. Numer. Meth. Engng. 37, 229-256.

2) Lu, Y. Y., Belytschko, T. and Tabbara, M. (1995). Element-free Galerkin method for wave propagation and dynamic fracture, Comput. Methods Appl. Mech. Engrg. 126, 131-153.

3) Jia, X. and Hu, T. (2006). Element-free precise integration method and its applications in seismic modeling and imaging, Geophys. J. Int., 166, 349-372.

4) Virieux, J. (1986). $P$-sv wave propagation in heterogeneous media: Velocity-stress finite difference method, Geophysics 51, 889-901

5) Graves, R. W. (1996). Simulating seismic wave propagation in 3D elastic media using staggered-grid finite differences, Bull. Seims. Soc. Am., 86, 1091-1106.

6) Jia, X. and Hu, T. (2006). Element-free precise integration method and its applications in seismic modeling and imaging, Geophys. J. Int., 166, 349-372.

7) Komatitsch, D. and Tromp, J. (1999) Introduction to the spectral element method for three-dimensional seismic wave propagation, Geophys. J. Int., 139, 806-822.

8) Dumbser, M. and Kaser, M. (2006) An arbitrary high-order discontinuous Galerkin method for elastic waves on isotropic unstructured meshes II. The three- dimensional isotropic case, Geophys. J. Int., 167, 319-336.

10) Collino, F. and Tsogka, C. (2001). Application of the perfectly matched absorbing layer model to the linear elastodynamic problem in anisotropic heterogeneous media, Geophysics 66, 294-307.

11) Festa, G. and Nielsen, S. (2003). PML Absorbing Boundaries, Bull. Seims. Soc. Am., 93, 891-903.

12) Saito, M. (1993). Branch line contribution in Lamb's Problem, Butsuri-Tnasa, 46, 372-380. (in Japanese) 

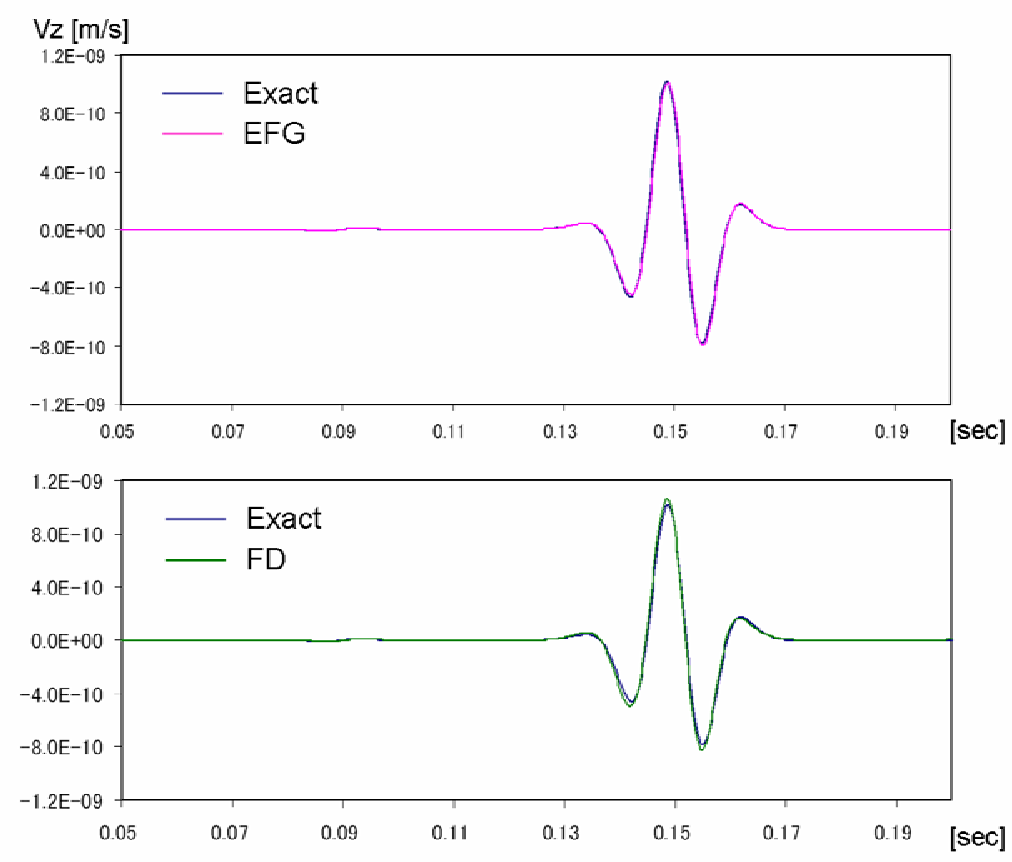

Figure.6 Comparison of the calculated waveforms for model A. EFG gives little more exact waveform than FD.
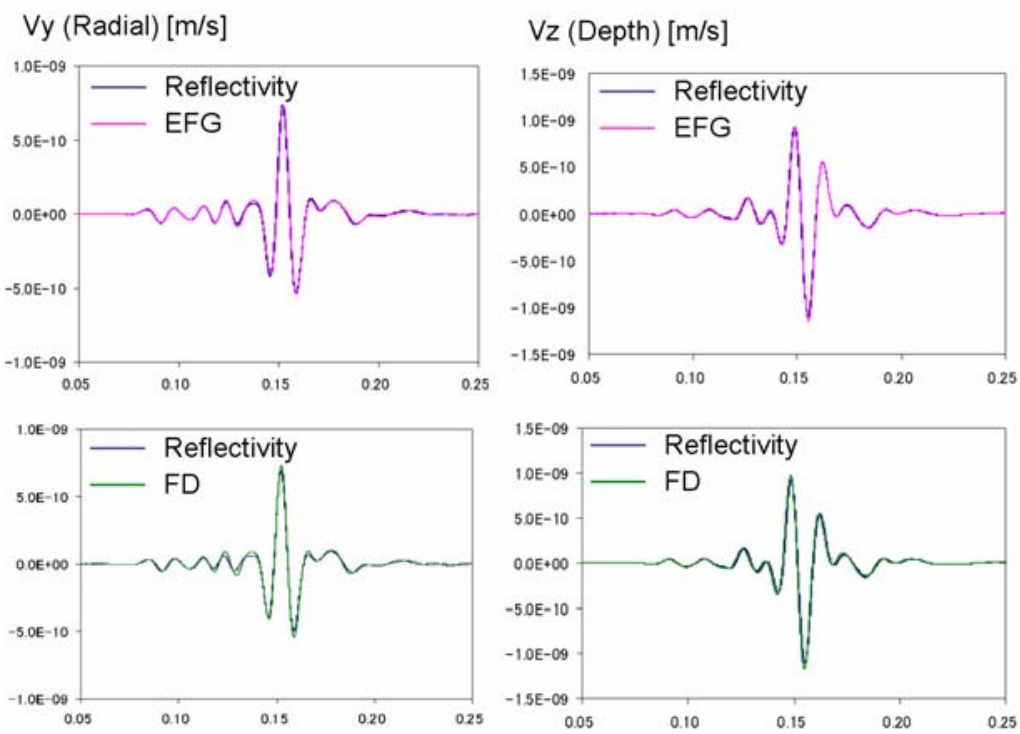

Figure.7 Comparison of the calculated waveforms for model B. EFG gives little more exact waveform than FD. 